\title{
The survival benefit and safety of No. 12a lymphadenectomy for gastric cancer patients with distal or total gastrectomy
}

\author{
Kun Yang ${ }^{1,2, *}$, Hai-Ning Chen ${ }^{1, *}$, Kai Liu ${ }^{1,2}$, Wei-Han Zhang ${ }^{1,2}$, Xin-Zu Chen ${ }^{1,2}$, \\ Xiao-Long Chen ${ }^{1,2}$, Zong-Guang Zhou ${ }^{1}$, Jian-Kun $\mathrm{Hu}^{1,2}$ \\ ${ }^{1}$ Department of Gastrointestinal Surgery, West China Hospital, Sichuan University, China \\ ${ }^{2}$ Laboratory of Gastric Cancer, State Key Laboratory of Biotherapy, West China Hospital, Sichuan University, China \\ * These authors contributed equally to this work \\ Correspondence to: Jian-Kun Hu, e-mail: hujkwch@126.com
}

Keywords: gastric cancer, gastrectomy, No.12a lymph nodes, survival, safety

Received: October 04, 2015

Accepted: January 29, 2016

Published: March 05, 2016

\section{ABSTRACT}

There has still not been a consensus in aspects of survival benefit and safety on No.12a lymph nodes (LNs) dissection for gastric cancer patients. This study was aimed to evaluate this issue for patients with distal or total gastrectomy. Patients were retrospectively divided into $12 \mathrm{aD}+$ group (with No.12a dissection) and 12aD-group (without No.12a dissection). Clinicopathologic characteristics, survival rate, morbidity and mortality were compared. There were 670 patients in $12 \mathrm{aD}+$ group, while 567 in 12aD-group. The baselines between the two groups were comparable. The No.12a LNs metastasis ratio was $11.6 \%$ and higher in lower third tumor. The metastasis of No.5 LNs, $\mathrm{N}$ stage and M stage were correlated to metastasis of No.12a LNs. There was no difference in morbidity nor mortality between the two groups. The 5-year overall survival rates (5-y OS) were $59.6 \%$ and $55.1 \%$ in $12 \mathrm{aD}+$ group and $12 \mathrm{aD}$-group respectively $(P=0.075)$. The $5-y$ OS of patients with negative and positive No.12a LNs were $62.3 \%$ and $24.1 \%$. The survival of stage III patients with No.12a positive was better than that of stage IV patients. The 5-y OS were better in 12aD+ group for patients with ages more than 60, lower third tumor, distal gastrectomy, N3 status, or III stages compared with 12aD-group. No.12a lymphadenectomy was independently better prognostic factors for stage III patients. No.12a LNs metastasis should not be considered as distant metastasis. No.12a lymphadenectomy can be performed safely and should be indicated for potentially curable progressive stage tumors requiring distal gastrectomy and might be reserved in patients with stage I or II, or upper third tumor.

\section{INTRODUCTION}

Gastric cancer is the fourth most common cancer and the second leading cause of cancer death worldwide, especially in East Asia [1]. Surgery is the mainstay of treatment for patients with gastric carcinoma and radical lymph nodes (LNs) dissection is an important part of curative resection. Controversy over the lymphadenectomy in the gastric cancer surgery has persisted for several decades. However, the benefiting role of standard D2 LNs dissection for the treatment of gastric cancer has been accepted by a majority of surgeons nowadays $[2,3]$. According to the Japanese gastric cancer treatment guideline (3rd English version) [4], D1 or D1+ lymphadenectomy could be performed only for T1N0M0 patients.
No. $12 \mathrm{a}$ LNs are defined as the LNs in the hepatoduodenal ligament along the hepatic artery [5]. No.12a LNs metastasis is considered as distant metastasis by 7 th American Joint Committee on Cancer (AJCC) classification recently since its significant poor prognosis compared to patients with metastases in the other extraperigastric nodes [6]. However, it is still considered as a regional LNs by the 3rd Japanese classification of gastric carcinoma and 6th AJCC classification, and should be dissected during D2 lymphadenectomy $[5,7]$. Actually, the dissection of No.12a LNs which was not included in a D2 lymphadenectomy for lower third gastric cancer according to the general rules for gastric cancer (1st English edition) was reassigned to the extent of D2 lymphadenectomy in the 2nd edition [8]. Even now, 
the definition of D2 lymphadenectomy in National Cancer Comprehensive Network (NCCN) guideline of gastric cancer (Version 3, 2015) does not include the No.12a LNs dissection [9]. Thus, the group of No.12a LNs has special distinctiveness.

In another hand, the survival benefit of No.12a lymphadenectomy is still controversial and not completely elucidated, although No.12a LNs are required to be dissected in D2 lymphadenectomy when distal or total gastrectomy is performed for advanced or $\mathrm{N}+$ tumors according to the Japanese guideline [4]. Moreover, No. 12a LNs dissection during standard D2 lymphadenectomy is not frequently performed in actual practice. The incidence of No.12a LNs metastasis has been reported from 9-13.1\% [10, 11]. The high metastatic incidence supports why No. 12a LNs should be removed in D2 dissection for gastric cancer. Meanwhile, some researches rebutted that No.12a LN metastasis regarded as distant metastasis was inappropriate and its dissection should be included in the extent of D2 lymphadenectomy [12, 13]. Nevertheless, some other researches argued that patients without No.12a lymphadenectomy would not compromise the survival, compared to standard D2 lymphadenectomy $[14,15]$. The therapeutic index of No. 12a LNs was only 2.7 for lower third tumor, and zero for upper third tumor [16-18].

Therefore, there still has not been a consensus in aspects of survival and safety on No.12a LNs dissection. The aim of this study is to evaluate the survival benefit and safety of No. 12a lymphadenectomy for gastric cancer patients with distal or total gastrectomy.

\section{RESULTS}

\section{Patient characteristics}

There were 670 patients in the $12 \mathrm{aD}+$ group and 567 in the $12 \mathrm{aD}-$ group. The baselines of two groups were comparable, including gender, age, comorbidity, tumor location, resection type, curative degree, differentiation, tumor size, depth of invasion, LNs metastasis, distant metastasis and staging (Table 1). There were 301 patients receiving chemotherapy in the $12 \mathrm{aD}+$ group and 248 in the $12 \mathrm{aD}$-group, without significant different $(P=0.676)$.

\section{Metastasis of No.12a LNs}

Because the analyses about the metastasis of No.12a LNs, including the metastatic ratio of No.12a LNs, the percentage of patients with positive No.12a LNs, and the correlations between the No.12a metastasis and clinicopathologic factors, didn't involve survival data and surgical data. In order to enroll more patients, we extended the study duration to the June, 2014 for the aforementioned analyses only.
There were totally 84 patients $(8.1 \%)$ with positive No.12a LNs in 1039 patients who underwent distal or total gastrectomy with No.12a lymphadenectomy. Totally 968 retrieved No.12a LNs with 112 involvement and the metastatic ratio was $11.6 \%(112 / 968)$. The results of metastasis of No.12a LNs according to different tumor locations are summarized in Table 2 . The tumor stages were more advanced in patients with positive No.12a LNs, ranking from stage IIIb to stage IV. Seventy four $(88.1 \%)$ of 84 patients with metastasis in the No.12a LNs had N3 disease.

Several clinicopathologic factors consisting of metastasis status of the No.3, No. 5, No. 7, No. 8a, No. 9 and No. 11p LNs, tumor location, tumor differentiation, tumor size, $\mathrm{T}$ stage, $\mathrm{N}$ stage and $\mathrm{M}$ stage were included in Logistic regression to analyze the correlations to metastasis of No.12a LNs. Results revealed that metastasis of No.5 LNs $(P=0.024)$, N stage $(P=0.005)$ and M stage $(P<0.001)$ were correlated to metastasis of No.12a LNs (Supplementary Table S1).

\section{Operative variables}

The mean number of harvested LNs was significantly higher in $12 \mathrm{aD}+$ group than that of $12 \mathrm{aD}-$ group at the cost of prolonged operation time $(P<0.001)$. There were no significant differences in the estimated blood loss $(P=0.109)$ and postoperative hospital stays $(P=0.418)$ between the two groups. There were 2 and 4 patients with reoperations in $12 \mathrm{aD}+$ and $12 \mathrm{aD}-$ groups respectively without statistically different $(P=0.422)$. The details can be seen in Table 3 .

\section{Morbidity and mortality}

The overall postoperative morbidity rates were $14.8 \%$ versus $11.8 \%$ in the $12 \mathrm{aD}+$ and $12 \mathrm{aD}$-groups without significant different $(P=0.128)$. Neither the constitution of severity of complications nor spectrum of postoperative complications between the two groups was significant different (Table 4). The postoperative mortality was $0.4 \%$ versus $0.3 \%$ in the $12 \mathrm{aD}+$ and $12 \mathrm{aD}-$ groups $(P=1.000)$. Three patients of $12 \mathrm{aD}+$ group and 2 patients of $12 \mathrm{aD}$-group died due to pulmonary failure, anastomotic leakage, and intraluminal hemorrhage.

\section{Long-term survival}

The 5-year overall survival (5-y OS) for patients with and without No. 12a lymphadenectomy were $59.6 \%$ and $55.1 \%$, respectively. Although the 5-y OS was slight better in the $12 \mathrm{aD}+$ group, this was not statistically significant $(P=0.075)$ (Figure 1$)$. Kaplan-Meier analysis showed that 5-y OS of patients with negative and positive No.12a LNs were $62.3 \%$ and $24.1 \%(P<0.001)$. Because the tumor stages in patients with positive No.12a LNs ranked from stage IIIb to stage IV, we compared the 5-y 
Table 1: General clinicopathologic characteristics of the patients

\begin{tabular}{|c|c|c|c|}
\hline & $\begin{array}{l}\text { 12aD+ group } \\
N=670(\%)\end{array}$ & $\begin{array}{l}\text { 12aD-group } \\
N=567(\%)\end{array}$ & $P$ value \\
\hline Gender & & & 0.078 \\
\hline Female & $199(29.7)$ & $195(34.4)$ & \\
\hline Male & $471(70.3)$ & $372(65.6)$ & \\
\hline Age (yrs) & & & 0.288 \\
\hline$<60$ & $390(58.2)$ & $313(55.2)$ & \\
\hline$\geq 60$ & $280(41.8)$ & $254(44.8)$ & \\
\hline Comorbidity & $325(48.5)$ & $300(52.9)$ & 0.123 \\
\hline Pulmonary & 135 & 120 & \\
\hline Digestive & 60 & 53 & \\
\hline Urological & 25 & 20 & \\
\hline Cardiovascular & 110 & 106 & \\
\hline Endocrinal & 70 & 64 & \\
\hline Neurological & 5 & 3 & \\
\hline Hematological & 12 & 9 & \\
\hline Longitudinal Tumor location & & & 0.169 \\
\hline Upper third & $79(11.8)$ & $46(8.1)$ & \\
\hline Middle third & $94(14.0)$ & $81(14.3)$ & \\
\hline Lower third & $481(71.8)$ & $429(75.7)$ & \\
\hline Whole stomach & $16(2.4)$ & $11(1.9)$ & \\
\hline Circumferential Tumor location & & & 0.109 \\
\hline Lesser curvature & $401(59.9)$ & $346(61.0)$ & \\
\hline Greater curvature & $88(13.1)$ & $61(10.8)$ & \\
\hline Anterior wall & $35(5.2)$ & $47(8.3)$ & \\
\hline Posterior wall & $56(8.4)$ & $51(9.0)$ & \\
\hline Full circle & $90(13.4)$ & $62(10.9)$ & \\
\hline Resection type & & & 0.072 \\
\hline Distal gastrectomy & $469(70.0)$ & $423(74.6)$ & \\
\hline Total gastrectomy & $201(30.0)$ & $144(25.4)$ & \\
\hline Curative degree & & & 0.394 \\
\hline R0 & $611(91.2)$ & $509(89.8)$ & \\
\hline $\mathrm{R} 1 / \mathrm{R} 2$ & $59(8.8)$ & $58(10.2)$ & \\
\hline Differentiation & & & 0.666 \\
\hline G1 & $14(2.1)$ & $17(3.0)$ & \\
\hline $\mathrm{G} 2$ & $96(14.3)$ & $70(12.3)$ & \\
\hline G3 & $560(83.6)$ & $480(84.7)$ & \\
\hline
\end{tabular}




\begin{tabular}{|c|c|c|c|}
\hline Tumor size $(\mathrm{cm})$ & & & 0.099 \\
\hline$\leq 2$ & $113(16.9)$ & $76(13.4)$ & \\
\hline$>2, \leq 5.0$ & $312(46.6)$ & $252(44.4)$ & \\
\hline$>5, \leq 8.0$ & $185(27.6)$ & $190(33.5)$ & \\
\hline$>8.0$ & $60(9.0)$ & $49(8.6)$ & \\
\hline Depth of infiltration $(\mathrm{T})$ & & & 0.771 \\
\hline $\mathrm{T} 1$ & $139(20.7)$ & $106(18.7)$ & \\
\hline $\mathrm{T} 2$ & $73(10.9)$ & $83(14.6)$ & \\
\hline T3 & $64(9.6)$ & $51(9.0)$ & \\
\hline $\mathrm{T} 4 \mathrm{a}$ & $334(49.9)$ & $264(46.6)$ & \\
\hline $\mathrm{T} 4 \mathrm{~b}$ & $60(9.0)$ & $63(11.1)$ & \\
\hline Nodal status $(\mathrm{N})$ & & & 0.770 \\
\hline N0 & $210(31.3)$ & $174(30.7)$ & \\
\hline N1 & $117(17.5)$ & $106(18.7)$ & \\
\hline $\mathrm{N} 2$ & $100(14.9)$ & $99(17.5)$ & \\
\hline N3a & $154(23.0)$ & $111(19.6)$ & \\
\hline $\mathrm{N} 3 \mathrm{~b}$ & $89(13.3)$ & $77(13.6)$ & \\
\hline Distal metastasis $(\mathrm{M})$ & & & 0.490 \\
\hline M0 & $592(88.4)$ & $508(89.6)$ & \\
\hline M1 & $78(11.6)$ & $59(10.4)$ & \\
\hline Stage & & & 0.574 \\
\hline Ia & $109(16.3)$ & 79 (13.9) & \\
\hline $\mathrm{Ib}$ & $49(7.3)$ & $51(9.0)$ & \\
\hline IIa & $40(6.0)$ & $42(7.4)$ & \\
\hline $\mathrm{IIb}$ & $84(12.5)$ & $80(14.1)$ & \\
\hline IIIa & $76(11.3)$ & $64(11.3)$ & \\
\hline $\mathrm{IIIb}$ & $78(11.6)$ & $66(11.6)$ & \\
\hline IIIc & $156(23.3)$ & $126(22.2)$ & \\
\hline IV & 78 (11.6) & $59(10.4)$ & \\
\hline
\end{tabular}

OS among patients with No.12a LNs negative/stage III, No.12a LNs positive/stage III, No.12a LNs negative/ stage IV and No.12a positive LNs/stage IV further. A significantly best 5-y OS was observed in patients with No.12a negative/stage III, compared to other three groups. The 5-y OS did not differ significantly between the patients with No.12a positive/stage IV and patients who had stage IV tumors without No.12a metastasis, both of which were worse than the survival of patients with No.12a positive/stage III (Figure 2).

When the subgroup analyses were performed stratified by clinicopathologic factors, the 5-y OS were better in $12 \mathrm{aD}+$ group for patients with ages more than $60(P=0.015)$, lower third tumor $(P=0.027)$, distal gastrectomy $(P=0.008), \mathrm{N} 3$ status $(P=0.036)$, or III stages $(P=0.026)$ compared to those of $12 \mathrm{aD}$-group (Figure 3 ). The better trend of $12 \mathrm{aD}+$ group also could be found in patients with T4 $(P=0.089)$ and patients with M0 $(P=0.051)$ although no significant differences (Figure 3, Supplementary Table S2). There were no significant differences of 5-y OS for other clinicopathologic factors between the two groups.

\section{Univariate and multivariate analyses for overall survival}

In stage I and II patients, depth of infiltration $(P=0.003)$ and LNs metastasis $(P=0.046)$ were 
Table 2: No.12a LNs metastasis according to longitudinal tumor location

\begin{tabular}{|l|c|}
\hline \multicolumn{1}{|c|}{ Metastasis of No.12 LNs } & Value \\
\hline Metastatic ratio & $6.1 \%(9 / 147)$ \\
\hline Upper third & $7.3 \%(8 / 110)$ \\
\hline Middle third & $13.1 \%(90 / 689)$ \\
\hline Lower third & $22.7 \%(5 / 22)$ \\
\hline Whole stomach & $11.6 \%(112 / 968)$ \\
\hline Total & \\
\hline Percentage of patients with positive No.12a LNs & $4.0 \%(7 / 177)$ \\
\hline Upper third & $6.1 \%(8 / 131)$ \\
\hline Middle third & $9.1 \%(64 / 704)$ \\
\hline Lower third & $18.5 \%(5 / 27)$ \\
\hline Whole stomach & $8.1 \%(84 / 1039)$ \\
\hline Total & \\
\hline
\end{tabular}

Table 3: Operative variables of the patients

\begin{tabular}{|l|c|c|c|}
\hline \multicolumn{1}{l}{} & $\begin{array}{c}\text { 12aD+ group } \\
(N=\mathbf{6 7 0})\end{array}$ & $\begin{array}{c}\text { 12aD-group } \\
(N=\mathbf{5 6 7})\end{array}$ & P value \\
\hline No. of harvested lymph nodes(mean \pm standard deviation) & $30.4 \pm 12.3$ & $25.8 \pm 12.2$ & $<0.001$ \\
\hline Postoperative days (mean \pm standard deviation) & $11.0 \pm 6.2$ & $11.3 \pm 7.1$ & 0.418 \\
\hline Estimated blood loss, $\mathrm{mL}$ (mean \pm standard deviation) & $157.6 \pm 139.0$ & $172.5 \pm 152.3$ & 0.109 \\
\hline Operation time, min(mean \pm standard deviation) & $248.2 \pm 49.8$ & $234.8 \pm 56.8$ & $<0.001$ \\
\hline No. of patients with reoperation (General anesthesia) & 2 & $4^{*}$ & 0.422 \\
\hline
\end{tabular}

* One patient received double valve replacements because of postoperative infectious endocarditis, rather than postoperative surgical-related complications.

identified as independently associated with survival after adjusting for the clinicopathologic factors (Table 5). And No.12a lymphadenectomy was not an independently survival associated factor. In stage III patients, depth of infiltration $(P=0.008)$, LNs metastasis $(P<0.001)$, curative degree $(P=0.004)$, No.12a lymphadenectomy $(P=0.037)$ and chemotherapy $(P<0.001)$ were identified as independently survival associated factors (Table 5).

\section{DISCUSSION}

The prognostic role of No.12a LNs metastasis and the impact of No. 12a lymphadenectomy on survival and operative safety for gastric cancer patients are still controversial. The previous researches focused on evaluating the difference of OS in patients with or without No.12a LNs metastasis, or compared the D1 or D1+ lymphadenectomy with D2 lymphadenectomy. To our limited knowledge, this is the first study to exclusively investigate the impact of No.12a lymphadenectomy on the survival.
In this research, our results have showed the metastasis ratio of No. 12a LNs was $11.6 \%$, which was in accordance with other researches [10,11, 19, 20]. And this data indicated that the dissection of No.12a LNs should be paid attention to. Logistic regression showed N stage and M stage were correlated to metastasis of No.12a LNs, which consisted with the finding that patients with positive No.12a LNs ranked from stage IIIb to stage IV and more than $85 \%$ of patients with positive No.12a LNs had N3 disease. Lee et al. reported $90 \%$ of patients with positive metastasis in the hepatoduodenal ligament LNs had N3 disease [13]. He et al. reported patients with No.12a LNs metastasis had extensive LNs involvement; N stage and $\mathrm{M}$ stage were independent predictors of No.12a LNs involvement $[12,21]$. In the addition, our results revealed that metastasis of No.5 LNs $(P=0.024)$ was also correlated to metastasis of No.12a LNs, which matched the lymphatic drainage flows of tumor and was supported by other study [12]. It has been reported that metastasis of No.12a LNs was correlated to the tumor location and 
Table 4: Morbidity and mortality

\begin{tabular}{|c|c|c|c|}
\hline & $\begin{array}{c}\text { 12aD+ group } \\
(N=670)\end{array}$ & $\begin{array}{c}\text { 12aD-group } \\
(N=567)\end{array}$ & $P$ value \\
\hline Number of patients with morbidity & $99(14.8 \%)$ & $67(11.8 \%)$ & 0.128 \\
\hline \multicolumn{4}{|l|}{ Clavien-Dindo classification } \\
\hline I & 31 & 23 & \\
\hline II & 46 & 21 & \\
\hline IIIa & 16 & 13 & \\
\hline IIIb & 2 & 4 & \\
\hline IVa & 0 & 4 & \\
\hline $\mathrm{IVb}$ & 1 & 0 & \\
\hline $\mathrm{V}$ & 3 & 2 & \\
\hline \multicolumn{4}{|l|}{ Surgical related complications } \\
\hline Digestive tract hemorrhage & 3 & 0 & 0.255 \\
\hline Intraperitoneal hemorrhage & 1 & 2 & 0.596 \\
\hline Digestive tract leakage & 2 & 5 & 0.257 \\
\hline Wound infection or dehiscence & 20 & 19 & 0.714 \\
\hline Intraperitoneal infection & 8 & 4 & 0.382 \\
\hline Intestinal obstruction & 3 & 0 & 0.255 \\
\hline Gastroparesis & 10 & 8 & 0.905 \\
\hline \multicolumn{4}{|l|}{ Non-surgical related complications } \\
\hline Pulmonary & 35 & 20 & 0.149 \\
\hline Renal & 0 & 1 & 0.458 \\
\hline Digestive & 9 & 3 & 0.145 \\
\hline Urinary & 3 & 4 & 0.709 \\
\hline Cardiac & 3 & 4 & 0.709 \\
\hline Endocrinal & 1 & 1 & 1.000 \\
\hline Hemostatic & 1 & 1 & 1.000 \\
\hline Other complications* & 3 & 3 & 1.000 \\
\hline Mortality & $3(0.4 \%)$ & $2(0.3 \%)$ & 1.000 \\
\hline
\end{tabular}

* including delirium, skin rash, tinnitus, vertigo, and arthritis.

\# due to pulmonary failure, anastomotic leakage, and intraluminal hemorrhage.

depth of invasion $[10,22]$. Although the frequencies of No.12a LNs metastasis were different according to the tumor locations, tumor location as well as T stage have not been identified as correlated factors in our study and some studies $[19,22]$. This discrepancy may partly be caused by relative small number of patients with positive No.12a LNs and number of positive No.12a LNs.

Our results showed the overall 5-y OS was slightly better in the $12 \mathrm{aD}+$ group without statistically different yet, which was in agreement with some researches $[14,15]$. But we should notice that there was no No.12a
LNs metastasis in stage I and II patients in present study. And it's reasonable that there was no significant different of OS for patients with relative early stage who have extreme low risk of No.12a LNs metastasis between the two groups. Consequently, the survival outcome of whole population would be biased. Therefore, we performed the subgroup analyses stratified by clinicopathologic factors. Our results showed the 5-y OS were better in $12 \mathrm{aD}+$ group for patients with lower third tumor, distal gastrectomy, N3 status, or stage III compared with $12 \mathrm{aD}$-group. In our and other studies, patients with distal third tumors or whole 


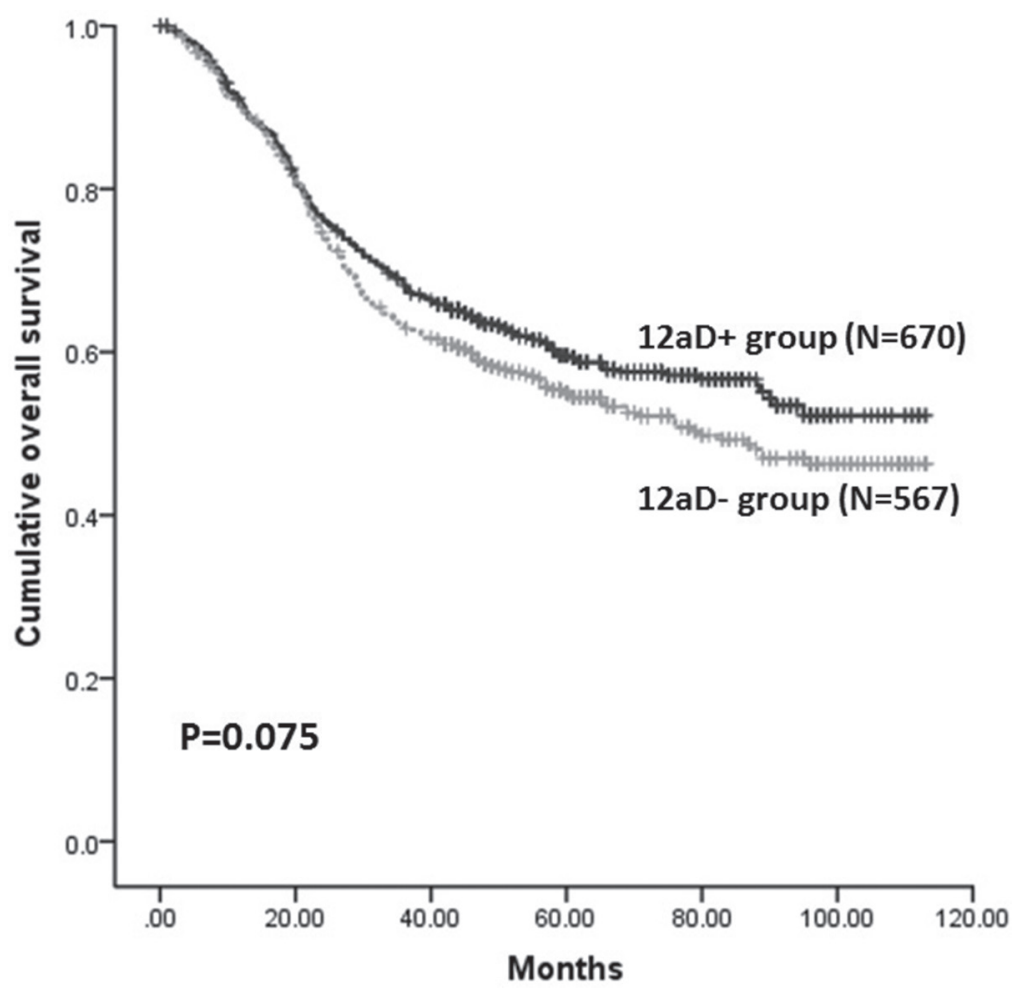

Figure 1: Kaplan-Meier survival analysis of patients between 12aD+ group and 12aD-group for overall patients $(P=0.075)$.

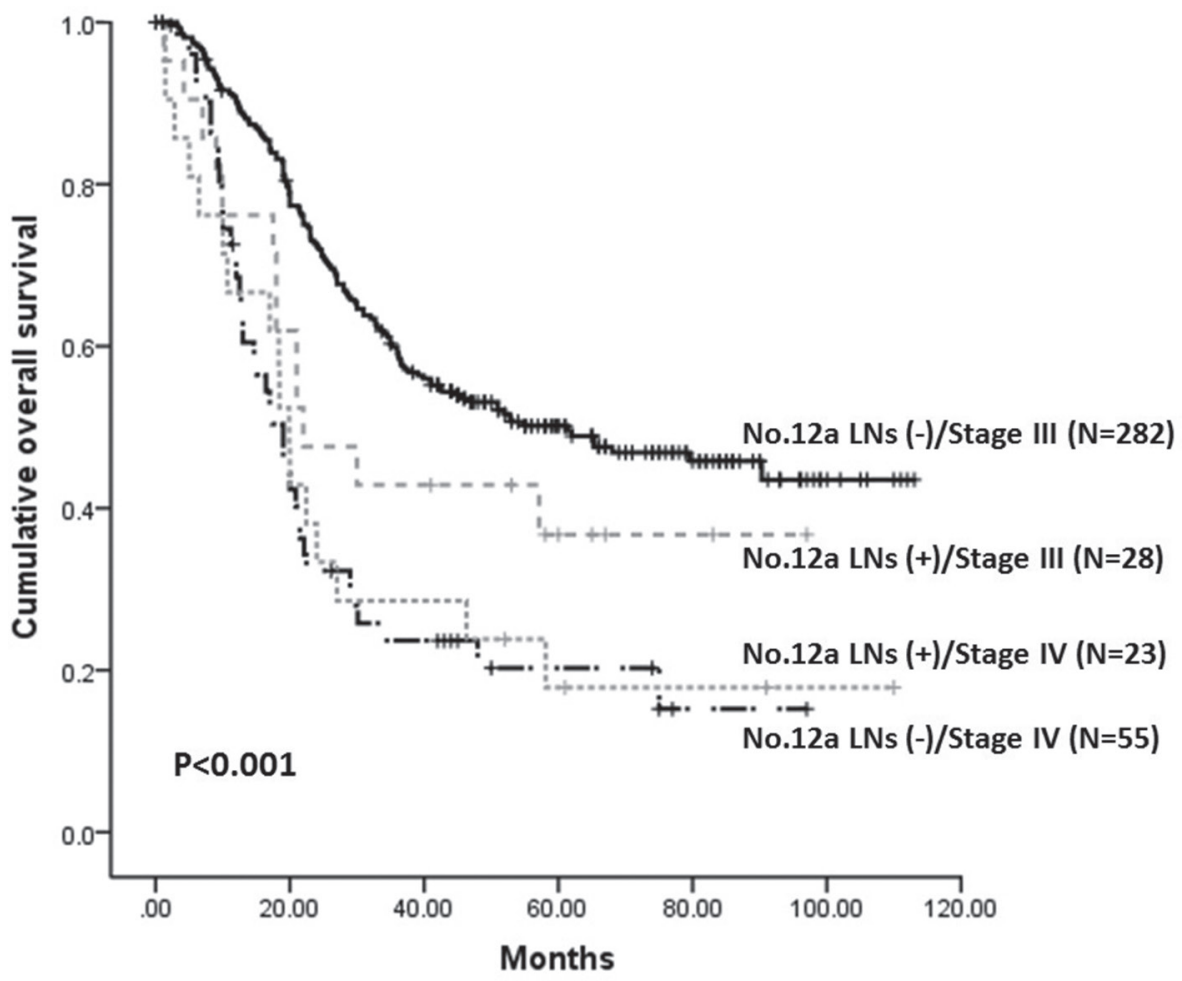

Figure 2: Kaplan-Meier survival analysis of patients with stage III/IV stratified by No.12a LNs metastatic status $(P<0.001)$. 
stomach lesion have relative more frequent No.12 LNs metastasis, whereas patients with upper third tumors have least $[16,23,24]$. This may be the reason why $12 \mathrm{aD}+$ group had showed significant better OS for patients with lower third tumor and distal gastrectomy. The fact that there was no significant difference of OS for patients with whole stomach lesion may be caused by the probable type II error. And as our study showed, the patients with positive No.12a LN ranked from stage IIIb to stage IV and most of them had N3 disease. These main partly explain the reason why there was a significant difference of OS for patients with N3 status, or III stages. Moreover, our results also indicated the dissection of No.12a LNs could still bring benefit to the patients even if the No.12a LNs were involved, regardless of stage IV patients. Roukos et al. reported D2 dissection had therapeutic value in patients with No.12a LNs metastases [25]. Multivariate analysis also proved that No.12a lymphadenectomy was independently better prognostic factors for stage III patients, rather than stage I/II patients. Based on the above results, we consider No.12a LNs dissection should be indicated for potentially curable progressive stage tumors requiring distal gastrectomy. Considering the low incidences of No. 12a LNs metastasis, No. 12a lymphadenectomy might be reserved in patients with stage
I or II gastric cancer or upper third tumor. The results should be confirmed further in well-designed randomized controlled trials (RCTs). Although comparison of survival between patients with and without No.12a LNs metastasis revealed that those with No.12a LNs metastasis had a significantly poorer survival outcome in our research, patients with distant metastasis had a significant worse overall survival than that of stage III patients with No.12a LN metastasis. Hence, we agree that No.12a LNs metastasis should not be considered as distant metastasis although No.12a LNs metastasis was an important indicator of poor prognosis $[12,13]$. However, this result come from a subgroup analysis and should be reviewed with more skepticism.

Although the number of examined LNs can be influenced by several factors, it was associated with the extent of lymphadenectomy [26, 27]. In present study, the mean number of harvested LNs was higher significantly in $12 \mathrm{aD}+$ group than that of $12 \mathrm{aD}$-group. Therefore, there was a concern that dissection of No.12a LNs may lead to a stage migration and could potentially account for survival differences. However, our results have showed that patients with positive No.12a LNs ranked from stage IIIb to stage IV. Most of patients with metastasis in the No.12a LNs had N3 disease. In another hand, the mean number of
A

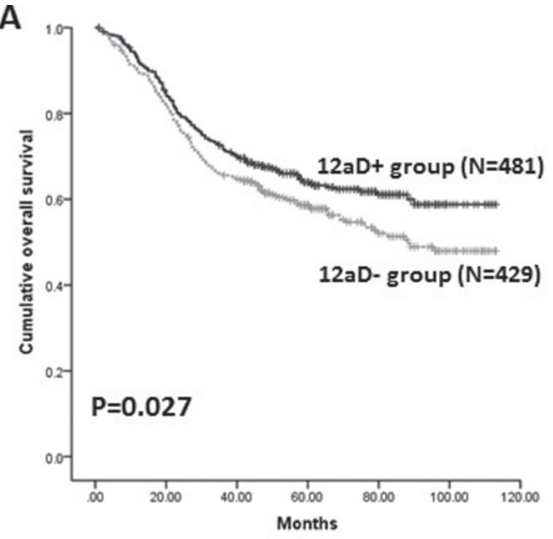

D

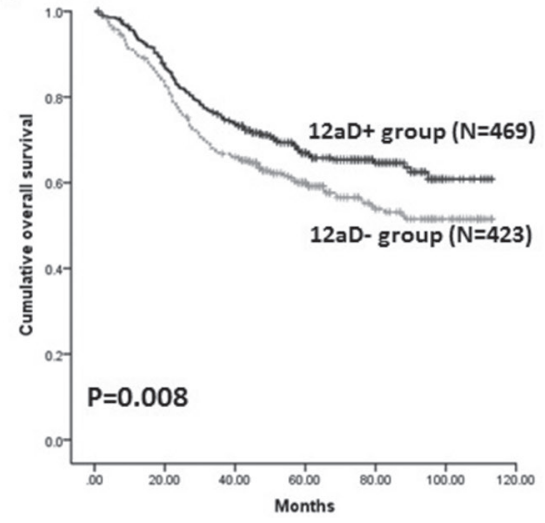

B

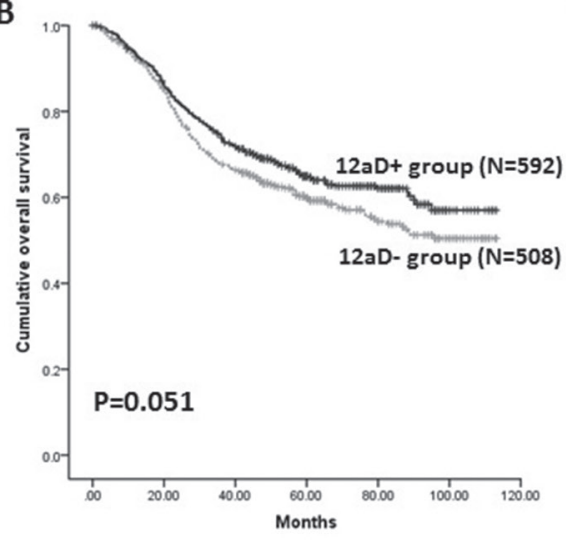

$\mathbf{E}$

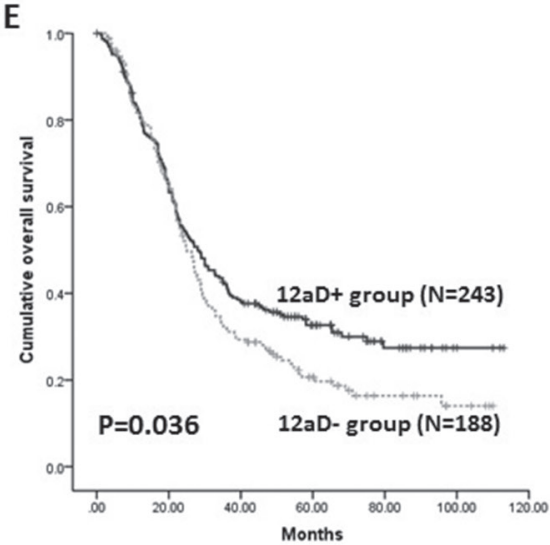

$C$

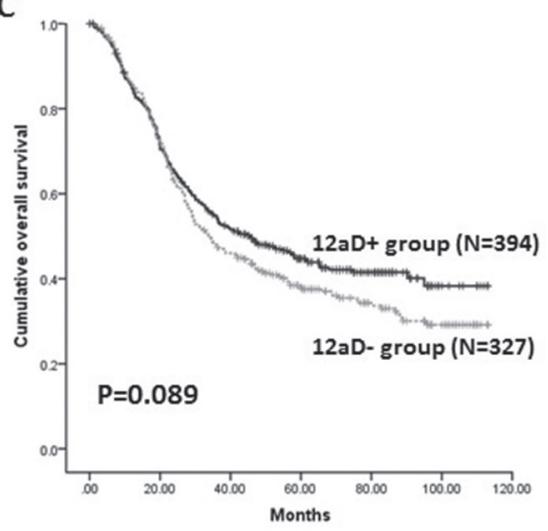

$\mathbf{F}$

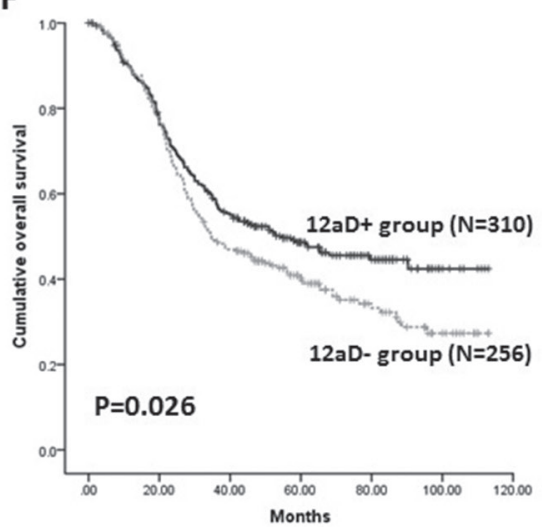

Figure 3: Kaplan-Meier survival analysis of patients between 12aD+ group and 12aD-group stratified by clinicopathologic factors. (A) Patients with lower third tumor $(P=0.027)$; (B) Patients with M0 $(P=0.051)$; (C) Patients with T4 $(P=0.089)$; (D) Patients with distal gastrectomy $(P=0.008)$; (E) Patients with N3 $(P=0.036)$; (F) Patients with stage III $(P=0.026)$. 
Table 5: Prognostic factors on the univariate and multivariate analysis

\begin{tabular}{|c|c|c|c|c|c|c|c|c|}
\hline & \multicolumn{4}{|c|}{ Stage I and Stage II patients } & \multicolumn{4}{|c|}{ Stage III patients } \\
\hline & $\begin{array}{c}\text { Univariate } \\
\text { HR } \\
(95 \% \mathrm{CI})\end{array}$ & $\begin{array}{c}P \\
\text { value }\end{array}$ & $\begin{array}{c}\text { Multivariate } \\
\text { HR } \\
(95 \% \text { CI })\end{array}$ & $P$ value & $\begin{array}{c}\text { Univariate } \\
\text { HR } \\
(95 \% \mathrm{CI})\end{array}$ & $P$ value & $\begin{array}{c}\text { Multivariate } \\
\text { HR } \\
(95 \% \text { CI })\end{array}$ & $P$ value \\
\hline Gender & & 0.891 & & & & 0.130 & & \\
\hline Male & 1 & & & & 1 & & & \\
\hline Female & $1.03[0.66-1.61]$ & 0.891 & & & $0.82[0.64-1.06]$ & 0.130 & & \\
\hline Age (yrs) & & 0.086 & & & & 0.066 & & \\
\hline$<60$ & 1 & & & & 1 & & & \\
\hline$\geq 60$ & $1.44[0.95-2.19]$ & 0.086 & & & $1.24[0.99-1.55]$ & 0.066 & & \\
\hline $\begin{array}{l}\text { Longitudinal Tumor } \\
\text { location }\end{array}$ & & 0.478 & & & & 0.289 & & \\
\hline Upper third & 1 & & & & 1 & & & \\
\hline Middle third & $1.36[0.36-5.12]$ & 0.653 & & & $0.75[0.50-1.12]$ & 0.158 & & \\
\hline Lower third & $1.80[0.57-5.69]$ & 0.320 & & & $0.78[0.57-1.07]$ & 0.120 & & \\
\hline Whole stomach & $-{ }^{*}$ & $-{ }^{*}$ & & & $1.16[0.55-2.45]$ & 0.699 & & \\
\hline $\begin{array}{l}\text { Circumferential } \\
\text { Tumor location }\end{array}$ & & 0.083 & & & & 0.085 & & \\
\hline Less curvature & 1 & & & & 1 & & & \\
\hline Great curvature & $0.75[0.38-1.48]$ & 0.412 & & & $1.12[0.78-1.61]$ & 0.548 & & \\
\hline Anterior wall & $1.75[0.94-3.27]$ & 0.080 & & & $1.00[0.60-1.67]$ & 0.997 & & \\
\hline Posterior wall & $0.65[0.26-1.63]$ & 0.361 & & & $1.49[1.00-2.21]$ & 0.049 & & \\
\hline Full circle & $1.98[0.90-4.35]$ & 0.089 & & & $1.46[1.07-2.00]$ & 0.019 & & \\
\hline Differentiation & & 0.396 & & & & 0.408 & & \\
\hline G1 & 1 & & & & $-{ }^{*}$ & $-{ }^{*}$ & & \\
\hline G2 & $2.20[0.51-9.49]$ & 0.290 & & & 1 & & & \\
\hline G3 & $2.53[0.62-10.34]$ & 0.196 & & & $0.86[0.55-1.34]$ & 0.408 & & \\
\hline Tumor size (cm) & & 0.147 & & & & 0.074 & & \\
\hline$\leq 2$ & 1 & & & & 1 & & & \\
\hline$\sim 5.0$ & $1.31[0.79-2.17]$ & 0.298 & & & $1.76[0.82-3.78]$ & 0.145 & & \\
\hline$\sim 8.0$ & $2.03[1.09-3.80]$ & 0.026 & & & $2.13[0.99-4.55]$ & 0.052 & & \\
\hline$>8.0$ & $0.75[0.10-5.55]$ & 0.776 & & & $2.41[1.07-5.45]$ & 0.034 & & \\
\hline $\begin{array}{l}\text { Depth of infiltration } \\
\text { (T) }\end{array}$ & & 0.005 & & 0.003 & & 0.040 & & 0.008 \\
\hline $\mathrm{T} 1$ & 1 & & 1 & & $-{ }^{*}$ & $-*$ & $-{ }^{*}$ & $-*$ \\
\hline $\mathrm{T} 2$ & $2.32[1.36-3.97]$ & 0.002 & 1.82 [1.01-3.29] & 0.046 & 1 & & 1 & \\
\hline $\mathrm{T} 3$ & $1.77[0.85-3.70]$ & 0.126 & 1.48 [0.69-3.18] & 0.312 & $0.74[0.36-1.50]$ & 0.398 & $0.95[0.46-1.95]$ & 0.881 \\
\hline $\mathrm{T} 4$ & $2.45[1.40-4.30]$ & 0.002 & $3.04[1.68-5.50]$ & 0.000 & $1.32[0.76-2.30]$ & 0.330 & $1.76[1.00-3.11]$ & 0.049 \\
\hline Nodal status (N) & & 0.045 & & 0.046 & & $<0.001$ & & $<0.001$ \\
\hline No & 1 & & 1 & & 1 & & 1 & \\
\hline N1 & $1.70[1.05-2.76]$ & 0.032 & $2.01[1.14-3.55]$ & 0.017 & $0.46[0.14-1.51]$ & 0.202 & $0.57[0.17-1.91]$ & 0.360 \\
\hline $\mathrm{N} 2$ & 2.15 [1.09-4.23] & 0.027 & $2.14[0.97-4.70]$ & 0.059 & $0.51[0.16-1.63]$ & 0.257 & $0.72[0.22-2.39]$ & 0.592 \\
\hline N3 & $1.93[0.47-7.93]$ & 0.362 & $2.93[0.69-12.36]$ & 0.144 & $1.15[0.37-3.61]$ & 0.805 & $1.73[0.53-5.60]$ & 0.361 \\
\hline
\end{tabular}




\begin{tabular}{|c|c|c|c|c|c|c|}
\hline Curative degree & & $-{ }^{*}$ & & $<0.001$ & & 0.004 \\
\hline R0 & 1 & & 1 & & 1 & \\
\hline $\mathrm{R} 1 / \mathrm{R} 2$ & $\because$ & $-*$ & $2.19[1.47-3.25]$ & $<0.001$ & $1.84[1.21-2.80]$ & 0.004 \\
\hline Resection type & & 0.926 & & 0.152 & & \\
\hline Subtotal & 1 & & 1 & & & \\
\hline Total & $1.03[0.55-1.94]$ & 0.926 & 1.18 [0.94-1.49] & 0.152 & & \\
\hline $\begin{array}{l}\text { No.12a } \\
\text { lymphadenectomy }\end{array}$ & & 0.527 & & 0.026 & & 0.037 \\
\hline Yes & 1 & & 1 & & 1 & \\
\hline No & $1.15[0.75-1.74]$ & 0.527 & $1.29[1.03-1.62]$ & 0.026 & $1.28[1.02-1.62]$ & 0.037 \\
\hline Chemotherapy & & 0.342 & & $<0.001$ & & $<0.001$ \\
\hline No & 1 & & 1 & & 1 & \\
\hline Yes & $0.81[0.52-1.25]$ & 0.342 & $0.63[0.50-0.79]$ & $<0.001$ & $0.60[0.47-0.75]$ & $<0.001$ \\
\hline
\end{tabular}

* No patients in this subgroup.

harvested No.12a LNs in the patients with positive No.12a LNs was $1.4 \pm 1.1$. In that case, it's very rare for the small mean number to influence the $\mathrm{N}$ stage of patients with more than 6 positive LNs. Actually, we have reanalyzed the stage of patients in the $12 \mathrm{D}+$ group provided that we did not count the number of positive No.12 LNs and found that there were only 3 patients downstaged of $\mathrm{N}$ stage without change of TNM staging. Even we re-compared the baselines between the two groups using the new stage. There was also no significant difference between the two groups. Therefore, the effect of stage migration caused by the dissection of No.12a LNs could be ignored.

With respect to the safety, our results failed to show that there were significant differences in morbidity and mortality between two groups, which was supported by other study [15]. Kitagawa et al. had reported 2 cases of hepatic infarction resulted from accidental injury of proper hepatic artery in gastric cancer operations [28]. However, No.12a lymphadenectomy related complications, such as injury of proper hepatic artery or portal vein, had not occurred in the two groups. In spite of Galizia et al. showed that patients undergoing total gastrectomy with modified D2 lymphadenectomy (without No.10, 11d and 12a LNs dissection) demonstrated a significant reduction of postoperative morbidity [14]. The routine performance of a splenectomy in this study may account for the increased morbidity in standard D2 group, rather than by the D2 lymphadenectomy itself [29]. Regarding the operation-related variables simultaneously, there were no significant differences in terms of estimated blood loss, length of hospital stay and reoperation rate between the two groups. Hence, we considered No.12a lymphadenectomy can be performed safely with low morbidity and mortality by experienced surgeon with adequate training.
As in any other retrospective studies, limitation of the current analysis includes possible selection bias, detection bias, and performance of analysis bias [30]. However, we have performed subgroup analyses and multivariate analysis to adjust for the shortcomings. In addition, probably there was type II error concerning some subgroup analysis (such as whole stomach subgroup). Anyway, large scale RCTs are needed to explore the survival benefit and safety of No.12a LNs dissection for gastric cancer patients.

In conclusion, No.12a LNs metastasis should not be considered as distant metastasis. No.12a lymphadenectomy can be performed safely and should be indicated for potentially curable progressive stage tumors requiring distal gastrectomy and might be reserved in patients with stage I or II, or upper third tumor.

\section{METHODS}

\section{Patients}

From January 2006 to December 2011, a total of 1237 patients with gastric carcinoma who underwent total or distal gastrectomy were retrospectively analyzed. Patients were divided into $12 \mathrm{aD}+$ group and $12 \mathrm{aD}$-group according to whether No. 12a LNs dissection was performed or not. The preoperative diagnosis of gastric carcinoma was confirmed by gastric endoscopy and biopsy. The exclusion criteria were as follows: (1) The patients with other kinds of gastric tumors rather than gastric adenocarcinoma, such as lymphoma, gastrointestinal stromal tumor or adenosquamous carcinoma. (2) Patients diagnosed with any previous malignancies. (3) Remnant gastric cancers. The West China Hospital research ethics committee approved retrospective analysis of anonymous data. 


\section{Surgical techniques}

In this study, all patients underwent distal or total gastrectomy with D2 or D2 (-No.12a) LNs dissection for gastric cancer [4]. The difference on the extent of lymph node resection was only No.12a LNs between the two groups. The controversial of No.12a lymphadenectomy existed in our institution during the study period. Consequently, although No.12a LNs are required to be dissected in D2 lymphadenectomy according to the Japanese guideline, some doctors did not dissect the No.12a LNs even if for advanced cases when they performed D2 dissection. Billroth I, Billroth II or Roux-en-Y anastomosis with mechanical stapler was performed to reconstruct the digestive tract. For No.12a lymphadenectomy, the anterior layer of hepatoduodenal ligament was opened firstly. After the dissection of No.5 LNs at the root of right gastric artery, the soft tissues which located anterior and interior to the proper hepatic artery were dissected up to the bifurcation of hepatic artery. Then these tissues were retracted leftward and the proper hepatic artery was retracted rightward which could create a surgical plane between the LNs bearing tissues and the proper hepatic artery. Then all the tissues were removed en-bloc along the surgical plane by a combination of blunt and sharp dissection until the exposure of anterolateral wall of the portal vein. Cautious were given to avoid the injury of blood vessels. All the operations were performed by expertise of surgeons specialized in gastrointestinal surgery, at the West China Hospital, Sichuan University.

\section{Follow-up}

Patients underwent a follow up which was done by telephone calls, letters, or outpatient visits. Followup assessments were performed every 3-6 months for the first 2 years, every $6-12$ months for $3-5$ years after surgery and then annually [31]. Fluoropyrimidine alone or fluoropyrimidine/platinum regimens were given to the patients who received postoperative chemotherapy. Overall survival was calculated from the time of surgery until death or the last follow-up for survived patients. As of June, 2015 , the overall follow-up rate was $91.4 \%$ (1131/1237).

\section{Clinicopathologic analysis}

The clinicopathologic features, such as gender, age, tumor size, tumor location, depth of tumor invasion, LNs metastasis, staging, morbidity, mortality, and survival outcome were collected from the prospective database and compared between $12 \mathrm{aD}+$ group and $12 \mathrm{aD}$-group. The complications were classified according to the Clavien-Dindo Classification [32]. Metastatic ratio of LNs was defined as the ratio of the number of metastatic LNs over the number of harvested LNs. Clinicopathologic terminology was based on the Japanese Classification of Gastric Carcinoma (3rd English version) [5].

\section{Statistical analysis}

SPSS 19.0 software (SPSS, Chicago, IL, USA) was used for statistical analyses. Variables of normality were tested, while confirming the normal distribution, where data are expressed as means \pm standard deviation. Two independent $t$-tests for quantitative data and Chisquare test or Fisher's exact test for categorical data were performed, or data was expressed as medians with a range taking the Spearman test into consideration. Survival curves were derived from Kaplan-Meier estimates, and the curves were compared by log-rank tests. The correlation between the No.12a metastasis and clinicopathologic factors was investigated by logistic regression with the forward stepwise (conditional) method. The multivariate regression was performed using the Cox proportion hazards model. Two-sided $p$ value less than 0.05 was considered as statistical significance.

\section{ACKNOWLEDGMENTS AND FUNDING}

This work was internal supported by Volunteer Team of Gastric Cancer Surgery (VOLTGA), West China Hospital, Sichuan University, P.R.China. Domestic support from (1) National Natural Science Foundation of China (No. 81301867, 81372344); (2) National High-Technology Research and Development Program ("863" Program) of China (2015AA020306); (3) Sichuan University Scholarship Fund.

\section{CONFLICTS OF INTEREST}

None.

\section{REFERENCES}

1. Jemal A, Bray F, Center MM, Ferlay J, Ward E, Forman D. Global cancer statistics. CA Cancer J Clin. 2011; 61:69-90.

2. Songun I, Putter H, Kranenbarg EM, Sasako M, van de Velde CJ. Surgical treatment of gastric cancer: 15-year follow-up results of the randomised nationwide Dutch D1D2 trial. Lancet Oncol. 2010; 11:439-449.

3. Sasako M, Sano T, Yamamoto S, Kurokawa Y, Nashimoto A, Kurita A, Hiratsuka M, Tsujinaka T, Kinoshita T, Arai K, Yamamura Y, Okajima K; Japan Clinical Oncology Group. D2 lymphadenectomy alone or with para-aortic nodal dissection for gastric cancer. N Engl J Med. 2008; 359: 453-462.

4. Japanese gastric cancer association. Japanese gastric cancer treatment guidelines 2010 (ver. 3). Gastric Cancer. 2011; 14:113-123.

5. Japanese Gastric Cancer Association. Japanese classification of gastric carcinoma: 3rd English edition. Gastric Cancer. 2011; 14:101-112. 
6. Edge SB, Byrd DR, Compton CC, Fritz AG, Greene FL, Trotti A. American Joint Committee on Cancer (AJCC) Cancer Staging Manual (7th edn). Springer: New York, 2010.

7. Greene FL, Page AL, Fleming ID, Fritz A, Balch CM, Haller DG, et al. American Joint Committee on Cancer: AJCC Cancer Staging Manual (6th edn). Springer: New York, 2002.

8. Japanese Research Society for Gastric Cancer. Japanese classification of gastric carcinoma, first English edition. Kanehara and Co, Ltd: Tokyo, 1995.

9. National Comprehensive Cancer Network. NCCN Clinical Practice Guidelines in Oncology (NCCN guidelines ${ }^{\circledR}$ ) Gastric Cancer Version3.2015. Available at http://www. nccn.org/professionals/physician_gls/PDF/gastric.pdf. Accessed April 15th, 2015.

10. Maruyama K, Gunvén P, Okabayashi K, Sasako M, Kinoshita T. Lymph node metastases of gastric cancer. General pattern in 1931 patients. Ann Surg. 1989; 210:596-602.

11. Kong SH, Yoo MW, Kim JW, Lee HJ, Kim WH, Lee KU, Yang HK. Validation of limited lymphadenectomy for lower-third gastric cancer based on depth of tumour invasion. Br J Surg. 2011; 98:65-72.

12. Shirong C, Jianhui C, Chuangqi C, Kaiming W, Xinhua Z, $\mathrm{Wu}$ S, Yulong H. Survival of proper hepatic artery lymph node metastasis in patients with gastric cancer: implications for d2 lymphadenectomy. PLoS One. 2015; 10:e0118953.

13. Lee SL, Lee HH, Ko YH, Song KY, Park CH, Jeon HM, Kim SS. Relevance of hepatoduodenal ligament lymph nodes in resectional surgery for gastric cancer. Br J Surg. 2014; 101:518-522.

14. Galizia G, Lieto E, De Vita F, Castellano P, Ferraraccio F, Zamboli A, Mabilia A, Auricchio A, De Sena G, De Stefano L, Cardella F, Barbarisi A, Orditura M. Modified versus standard D2 lymphadenectomy in total gastrectomy for nonjunctional gastric carcinoma with lymph node metastasis. Surgery. 2015; 157:285-296.

15. Ichikura T, Chochi K, Sugasawa H, Mochizuki H. Modified radical lymphadenectomy (D1.5) for T2-3 gastric cancer. Langenbecks Arch Surg. 2005; 390:397-402.

16. Sasako M, McCulloch P, Kinoshita T, Maruyama K. New method to evaluate the therapeutic value of lymph node dissection for gastric cancer. Br J Surg. 1995; 82:346-351.

17. Goto H, Tokunaga M, Miki Y, Makuuchi R, Sugisawa N, Tanizawa Y, Bando E, Kawamura T, Niihara M, Tsubosa Y, Terashima M. The optimal extent of lymph node dissection for adenocarcinoma of the esophagogastric junction differs between Siewert type II and Siewert type III patients. Gastric Cancer. 2015; 18:375-381.

18. Yamashita H, Katai H, Morita S, Saka M, Taniguchi H, Fukagawa T. Optimal extent of lymph node dissection for Siewert type II esophagogastric junction carcinoma. Ann Surg. 2011; 254:274-280.
19. Keller E, Stützer H, Heitmann K, Bauer P, Gebbensleben B, Rohde H. Lymph node staging in 872 patients with carcinoma of the stomach and the presumed benefit of lymphadenectomy. German Stomach Cancer TNM Study Group. J Am Coll Surg. 1994; 178:38-46.

20. Wei ZW, Xia GK, Wu Y, Schwarz RE, Smith DD, He YL, Zhang $\mathrm{CH}$. Evaluation of skeletonization of the hepatoduodenal ligament for the lower third gastric cancer by propensity score analysis. Hepatogastroenterology. 2013; 60:1789-1796.

21. $\mathrm{Wu} \mathrm{H}, \mathrm{Wu} \mathrm{WH}, \mathrm{Xu} J \mathrm{~B}$, Zhang $\mathrm{XH}$, Wang L, Ma JP, Chen CQ, Cai SR, He YL, Zhan WH. Risk factors and prognostic impact of No.12 lymph node metastasis in cases with curable advanced distal gastric cancer. [Article in Chinese]. Zhonghua Yi Xue Za Zhi. 2013; 93:3847-3851.

22. Di Leo A, Marrelli D, Roviello F, Bernini M, Minicozzi A, Giacopuzzi S, Pedrazzani C, Baiocchi LG, de Manzoni G. Lymph node involvement in gastric cancer for different tumor sites and T stage: Italian Research Group for Gastric Cancer (IRGGC) experience. J Gastrointest Surg. 2007; 11:1146-1153.

23. Wu CW, Hsieh MJ, Lo SS, Tsay SH, Lui WY, P'eng FK. Lymph node metastasis from carcinoma of the distal onethird of the stomach. Cancer. 1994; 73:3109-3114.

24. Wang LS, Wu CW, Hsieh MJ, Fahn HJ, Huang MH, Chien KY. Lymph node metastasis in patients with adenocarcinoma of gastric cardia. Cancer. 1993; 71: 1948-1953.

25. Roukos DH, Lorenz M, Encke A. Evidence of survival benefit of extended (D2) lymphadenectomy in western patients with gastric cancer based on a new concept: a prospective long-term follow-up study. Surgery. 1998; 123:573-578.

26. Coburn NG, Swallow CJ, Kiss A, Law C. Significant regional variation in adequacy of lymph node assessment and survival in gastric cancer. Cancer. 2006; 107: 2143-2151.

27. Yang K, Zhang WH, Hu JK. Lymph Node Count as a Quality Measure for Gastric Cancer Surgery. JAMA Surg. $2015 ; 150: 595-596$.

28. Kitagawa T, Iriyama K. Hepatic infarction as a complication of gastric cancer surgery: report of four cases. Surg Today. 1998; 28:542-546.

29. Yang $\mathrm{K}$, Zhang WH, Chen XZ, Hu JK. Comparison of modified D2 lymphadenectomy versus standard D2 lymphadenectomy in total gastrectomy for gastric cancer patients with lymph nodes involvement. Surgery. 2015; 158:1446-7. doi: 10.1016/j.surg.2015.03.010.

30. Yang K, Zhang WH, Chen XZ, Chen XL, Zhang B, Chen ZX, Zhou ZG, Hu JK. Survival benefit and safety of no. 10 lymphadenectomy for gastric cancer patients with total gastrectomy. Medicine (Baltimore). 2014; 93:e158. 
31. Ajani JA, Bentrem DJ, Besh S, D'Amico TA, Das P, Denlinger C, Fakih MG, Fuchs CS, Gerdes H, Glasgow RE, Hayman JA, Hofstetter WL, Ilson DH, et al. National Comprehensive Cancer Network. Gastric cancer, version 2.2013: featured updates to the NCCN Guidelines. J Natl Compr Canc Netw. 2013; 11:531-546.
32. Clavien PA, Barkun J, de Oliveira ML, Vauthey JN, Dindo D, Schulick RD, de Santibañes E, Pekolj J, Slankamenac K, Bassi C, Graf R, Vonlanthen R, Padbury R, et al. The Clavien-Dindo classification of surgical complications: fiveyear experience. Ann Surg. 2009; 250:187-196. 